\title{
Expression, identification and biological effects of the novel recombination protein, PACAP38-NtA, with high bioactivity
}

\author{
LUSHENG WU, JING WANG, XIAOJIA CHEN and AN HONG \\ Biomedicine Institute, College of Life Science and Technology, Jinan University, \\ Guangdong Provincial Key Laboratory of Bioengineering Medicine, \\ National Engineering Research Center of Genetic Medicine, \\ Guangzhou, Guangdong 510632, P.R. China
}

Received July 24, 2014; Accepted November 7, 2014

DOI: $10.3892 / \mathrm{ijmm} .2014 .2017$

\begin{abstract}
Pituitary adenylate cyclase-activating polypeptide (PACAP) is a type of neuropeptide with multiple biological functions. However, it has a short half-life period in the body, $\sim 3$ to $5 \mathrm{~min}$, restricting its further development as a drug that can promote the recovery of nerve injury. In vitro and in vivo experiments have shown that PACAP can repair the epithelial cell on the surface of the injured cornea, as PACAP can act on the trigeminal nerve cell to secrete other active neurotransmitters, which can promote corneal epithelial cell proliferation and differentiation. In the present study, PACAP is connected to the $\mathrm{N}$-terminal agrin domain $(\mathrm{NtA})$ with a genetic engineering method, which allows the function of repairing the injured nerve. Notably, the recombinant polypeptide can interact with laminin, improving the biological effect of PACAP in repairing the injured nerve. In the study, the recombinant protein was constructed by combining PACAP38 and NtA by genetic engineering, and it is expressed in the pronucleus escherichia coli. The recombinant protein, PACAP38-NtA, is obtained with a two-step purification method, including anion-exchange chromatography and Ni-affinity chromatography, with the purity reaching $>90 \%$. The in vitro experiment has shown that this recombinant protein not only has the neurotrophy and neural restoration function of PACAP, but also has the function of an anchoring protein as laminin interacts with NtA. According to the in vitro anti-apoptosis, PC12 axon growth and ELISA experiments, this protein has the biological activity of a recombinant protein. PACAP38-NtA also has an anchoring function as NtA and laminin interact with good biological activity.
\end{abstract}

Correspondence to: Professor An Hong, Biomedicine Institute, College of Life Science and Technology, Jinan University, Guangdong Provincial Key Laboratory of Bioengineering Medicine, National Engineering Research Center of Genetic Medicine, 601 West Road of Huangpu, Guangzhou, Guangdong 510632, P.R. China

E-mail: tha@jnu.edu.cn

Key words: PACAP38, recombination protein, neuropeptide, $\mathrm{NtA}$

\section{Introduction}

The pituitary adenylate cyclase-activating polypeptide (PACAP) is a polypeptide with various biological activities that was discovered in the study by Miyata et al (1), which was investigating the hypothalamic hypophysiotropic hormone in 1989 (1-5) and it is also a new member in the family of secretin/glucagon/vasoactive intestinal peptide (VIP) (6-10). According to the previous studies, PACAP exists in the body in two forms; PACAP38 with 38 types of amino acid and PACAP27 with 11 types of amino acid but without the C-terminus (11-15). PACAP and its receptor are distributed in the central and peripheral nervous systems, as well as the surrounding tissues and organs, such as the pancreas, pancreas islet, digestive tract and genital glands $(7,16,17)$.

PACAP mainly has three types of receptors, which are PAC1R, VPAC1R and VPAC2R. The first is the main acceptor of PACAP, whereas the other two are the common acceptors of PACAP and VIP $(3,7,18)$. The three types of receptors are widely spread in the cardiovascular, respiratory, genital and nervous systems $(3,7,19)$ The content of $\mathrm{PAC1R}$ in the central nervous system is higher compared to VPAC1R and VPAC2R, and PAC1R spreads wider than the other two in central nervous system $(7,20,21)$. Studies have shown that PACAP promotes the repairing of injured nervous tissue $(20,22)$, as well as the differentiation of embryonic stem cells into neurons (23). During this process, PAC1R acceptor expression increases and PAC1R also improves the survival amount of neurons following spinal cord compression (24-28). In the eyes, PACAP and its acceptor are mainly distributed in the cornea, iris, Schacher's ganglion, choroid membranes and retina $(7,29)$. The study by Wang et al $(30)$ discovered that in the inflammatory responses of the ocular surface, PACAP, as the neurotransmitter of C-fibers, plays a positive feedback regulation role in the release of inflammatory factors from C-fibers. PACAP and its main acceptor PAC1 are widely spread in the eyes. PACAP is nutritious, and it can repair the nerve and also promote the repair of the corneal epithelium and regulation of the ocular inflammatory reaction. Therefore, it plays a vital role in the neural restoration and the recovery of cornea sensory following corneal flap surgery. 
$\mathrm{N}$-terminal agrin domain $(\mathrm{NtA})$ is the receptor protein of laminin, which is a type of proteoglycan that exists in the extracellular matrix, (ECM) and was firstly obtained following separation from the ECM of the Torpedo electric organ synapses by $\mathrm{Fu}$ and Gordon (31). The C-terminal structural domain of agrin covers all the regions that may interact with the surface of the skeletal muscle, particularly the three independent spherical G-shaped structural regions, and it can also induce the congregation of acetylcholine receptors, similar to the full-length agrin (32). Therefore, only acting as an 'anchoring region', the NtA of agrin allows its functional region, which is the combination of C-terminal with other specific parts, to perform its biological function. In 2009, Sun et al (33) reported that a fusion protein [known as LBD-nerve growth factor (NGF)] had been constructed by combining the NtA of agrin with NGF, and the fusion protein promotes the effect of repairing nerve regeneration.

In the present study, the C-terminal of PACAP38 and NtA were connected through a linker peptide (including 16 types of amino acid), constructing the fusion protein PACAP38-NtA. Theoretically, the recombination polypeptide has the biological activity of PACAP, and it can be combined with laminin to improve the remediation efficiency of the PACAP polypeptide. Furthermore, it can avoid the cutting of the C-terminal from PACAP38 by carboxypeptidase, thus preventing the decrease of receptor-binding capacity. In addition, it shows that the Schwann cells aggregate in the nerve injury, and significantly express laminin, indicating that the polypeptide can combine the cells in the neural injury and improve the effect of injury repair.

\section{Materials and methods}

Expression and purification. The structure of the recombinant DNA segment of PACAP38-NtA that was applied for the patent of invention (ID: 201310057657.7) is shown in (Fig. 1A). The DNA segment was chemically synthesized by GenScript, Inc. (Piscataway, NJ, USA). Following PCR amplification, the target segment was transferred into the carrier pET-3c, obtaining the cloning vector bacteria of the recombinant DNA segment. Subsequently, it was transferred to expression bacteria BL21 (DE3), achieving enough expression bacteria with the protein of interest after inducing with $1 \mathrm{mmol} / \mathrm{l}$ isopropylthiogalactoside (IPTG) at $30^{\circ} \mathrm{C}$ for $4 \mathrm{~h}$. The protein of interest was obtained through crushing bacteria and centrifugation was purified by the two-step purification; anion-exchange chromatography and Ni-column affinity chromatography. The purity was tested with SDS-PAGE analysis, western blotting detection for the PACAP antibody [rabbit anti-human PACAP38, 1:1000, Sigma, St. Louis, MO, USA; goat anti-rabbit immunoglobulin G (IgG) (heavy and light), 1:3000, Cell Signaling Technology, Danvers, MA, USA] and high-performance liquid chromatography (HPLC), and its molecular weight was tested with mass spectrometry.

Cell culture and research methods. PC12 cell (Committee on Type Culture Collection of Chinese Academy of Sciences, Shanghai, China) was cultured in RPMI-1640 medium with $10 \%$ fetal bovine serum (FBS) in a humidified atmosphere containing $5 \% \mathrm{CO}_{2}$. In total, $8 \times 10^{3}$ cells/holes were added to a 96 -hole plate, and the peripheral 36 holes were sealed with phosphate-buffered saline (PBS). Starvation medium with $0.5 \%$ FBS was employed after the cells were 50-60\% confluent, and subsequently $100 \mathrm{nM}$ of PACAP38-NtA protein were added and sterilized with a $0.22-\mu \mathrm{m}$ filter. The hole without PACAP-NtA was the negative control, while the wild-PACAP was taken as the positive control. After cultivating for $24 \mathrm{~h}, 10 \mu \mathrm{l}$ cell counting kit-8 (CCK8) reagent was added into each hole. After cultivating for $1 \mathrm{~h}$, the absorbance value was measured with $450 / 630 \mathrm{~nm}$ dual wavelength on the enzyme mark instrument (Bio-Rad, Hercules, CA, USA), and the growth curve was created with concentration and absorbance on the $\mathrm{X}$ and $\mathrm{Y}$ axis, respectively.

Quantitative analysis experiment of neurite. Smit et al (34) reported the experiment of analyzing the nervous processes for PC12 cells with quantitative analysis. Assays were performed in 24-well dishes (Corning Life Sciences, Tewksbury, MA, USA). Fresh transwell cell culture inserts were placed in wells containing ECM protein solutions (propagation, $10 \mu \mathrm{g} / \mathrm{ml}$ ) and incubated for $2 \mathrm{~h}$ at $37^{\circ} \mathrm{C}$. The insert was removed and transferred to a new well containing PACAP and PACAP-NtA with different concentrations. After $24 \mathrm{~h}$, the nervous processes were dyed purple by crystal violet subsequent to crossing the transwell insert. Images of $100 \mathrm{nM}$ polypeptide at different time points $(0,3,6,12$ and $24 \mathrm{~h})$ were captured to observe the dyeing effect, and subsequently the nervous processes that were dyed in purple were dissolved with glacial acetic acid, and the absorbance value at $570 \mathrm{~nm}$ was detected.

ELISA. The ELISA detection of the laminin protein was purchased from Sigma to test the combination ability of PACAP38-NtA. An appropriate amount of laminin was covered on 96 ELISA plates (PerkinElmer, Inc., Waltham, MA, USA), maintaining at $4^{\circ} \mathrm{C}$ for $24 \mathrm{~h}$ and discarding the liquid. Subsequently, the plate was dried and washed with PBS (pH 7.3) three times. Bovine serum albumin [2.5\% (w/v)], including $0.1 \%(\mathrm{v} / \mathrm{v})$ Tween 20 , was added into each hole ( $200 \mu \mathrm{l} /$ hole) and incubated at $37^{\circ} \mathrm{C}$ for $2 \mathrm{~h}$ for the blocking reaction. PACAP38-NtA at a concentration of $100 \mathrm{nM}$ was added into the enzyme labeling hole $(100 \mu \mathrm{l} / \mathrm{hole})$ at $37^{\circ} \mathrm{C}$ for $2 \mathrm{~h}$. PBS was used as the negative control, while $100 \mathrm{nM}$ wild-PACAP was the positive control. The proteins that did not combine were eliminated subsequent to washing with PBS and Tween 20 (PBST) three times. The rabbit anti-human PACAP38 antibody (1:1000; Sigma) and goat anti-rabbit IgG-HRP (1:1000) were added and maintained at $37^{\circ} \mathrm{C}$ for $1 \mathrm{~h}$, washing with PBST three times. The 3,3',5,5'-tetramethylbenzidine substrate was added (200 $\mu \mathrm{l} /$ hole) and incubated at room temperature for $10 \mathrm{~min} ; 2 \mathrm{M} \mathrm{H}_{2} \mathrm{SO}_{4}$ stopped coloration and absorbance was detected at $450 \mathrm{~nm}$.

Western blot analysis. Following electrophoresis, the protein blots were transferred to a PVDF membrane. The membrane was blocked with 5\% skimmed milk in TBST and incubated with primary antibody in TBST containing $2 \%$ skimmed milk overnight at $4^{\circ} \mathrm{C}$. After washing three times with TBST, the membrane was incubated at room temperature for $1 \mathrm{~h}$ with secondary antibody diluted with TBST containing $2 \%$ skimmed milk. The detected protein signals were visualized by an electrochemiluminescence system. 


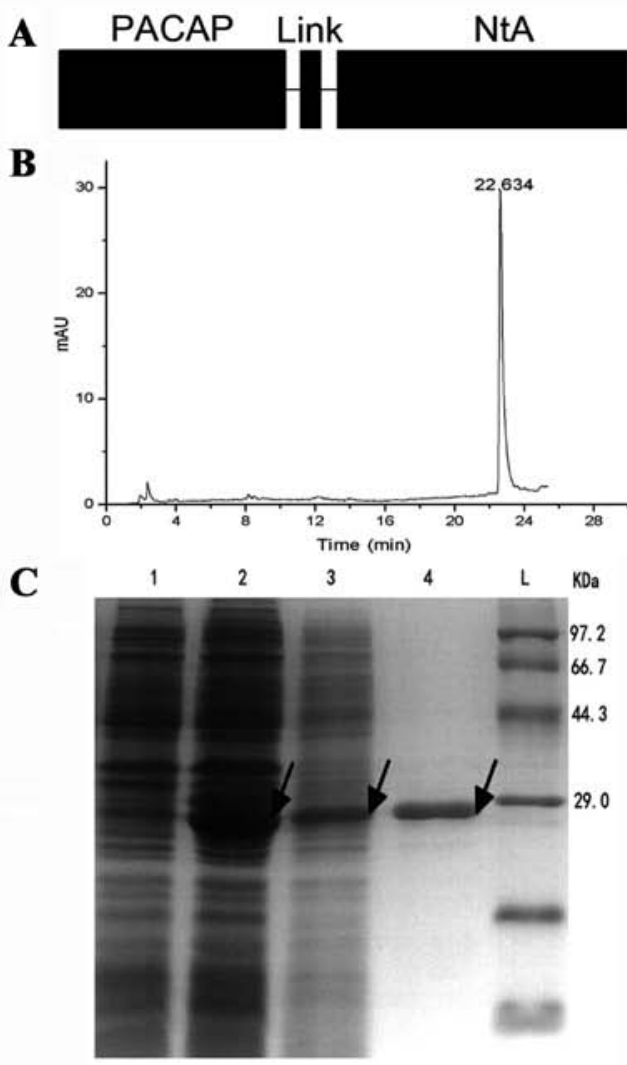

D Applied Biosystems 4700 Proteomics Analyzer 208

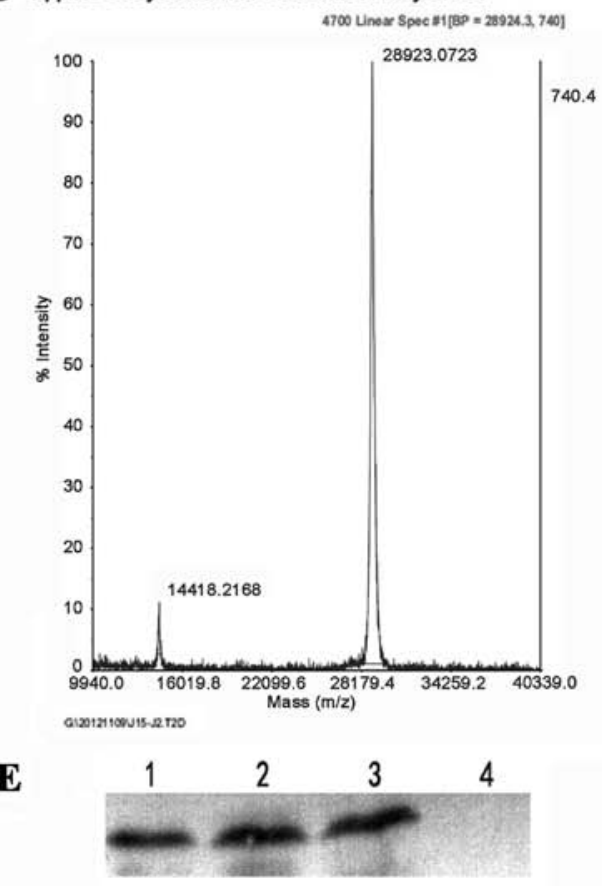

Figure 1. Expression, purification and identification of PACAP38-NtA. (A) Structural diagram of the recombinant protein. (B) The results of PACAP38-NtA expression and purification. L, protein ladder; 1 , the expressed result of PACAP38-NtA without IPTG; 2, the expressed result of PACAP38-NtA by IPTG; 3 , the supernatant containing PACAP38-NtA prior to purification; 4, the result of PACAP38-NtA subsequent to purification. (C) The high-performance liquid chromatography results of PACAP38-NtA. (D) The results of PACAP38-NtA at mass spectrometric analysis. (E) Detection of PACAP38-NtA by western-blotting analysis. 1, the expressed PACAP38-NtA by IPTG; 2 , the supernatant containing PACAP38-NtA prior to purification; 3, NtA-APCAP subsequent to purification; 4, the expressed E. coli. BL21 (DE3) by IPTG, as control. PACAP, pituitary adenylate cyclase-activating polypeptide; $\mathrm{NtA}, \mathrm{N}$-terminal agrin domain; IPTG, isopropylthiogalactoside.
Surgical procedures and application of PACAP38-NtA. Six eight-month-old C57 mice purchased from the Laboratory Animal Center of Sun Yat-sen University in China were injected with $7 \mu \mathrm{l} 10 \%$ chloral hydrate through the abdomen for anesthesia. A circular injury was created by a scratch on the cornea with the mini-keratome system (MK-2000; Nidek, Inc., Fremont, CA, USA) under the anatomical lens, which was $\sim 2 \mathrm{~mm}$ in diameter. The injury was dyed green with $2 \%$ sodium fluorescein and images were captured and labeled as $0 \mathrm{~h}$. The mice were randomly divided into three groups by adding $5 \mu \mathrm{l}$ normal saline, $5 \mu 1100 \mathrm{nM}$ PACAP38-NtA and $100 \mathrm{nM}$ wild PACAP, respectively. The repairing of the injury after 12,18 , 24 and $36 \mathrm{~h}$, respectively, was observed with the images. The images were captured and the repairing time of corneal injury in the different groups was recorded. All the animals were treated in accordance with the ARVO Statement for the Use of Animals in Ophthalmic and Vision Research.

Statistical analysis. Statistical analyses were performed using Excel 2003 software. Data obtained from three or more separate experiments are expressed as the means \pm standard deviation. Data were compared using standard analysis of variance methodology for repeated measurement and calculation of P-values. Differences were considered to indicate statistical significance at the $5 \%$ level $(\mathrm{P}<0.05)$.

\section{Results}

Obtaining the protein of interest. Following the construction of the PACAP38-NtA prokaryotic expression vector with molecular cloning, the soluble protein was obtained through IPTG inducible expression when the bacteria OD600 was between 0.4 and 0.6. Subsequently, the supernatant was collected after crushing the bacteria and centrifugation, and the protein of interest was obtained with the two-step purification method (Fig. 1C) and its purity was confirmed with HPLC (Fig. 1B). According to the western blotting PACAP38-NtA analysis of the rabbit anti-human PACAP38 antibody (Fig. 1E), the protein of interest was expressed, with a purity $>90 \%$, and its molecular weight was confirmed by mass spectrometry as $28.9 \mathrm{kDa}$ (Fig. 1D), which was in accordance with the molecular weight of SDS-PAGE electrophoresis (Fig. 1C). These preliminary results indicated that the target protein was expressed and purified, and could be activated in the subsequent experiments.

Binding capacity of PACAP39-NtA. The detection of the antigen antibody reaction with ELISA had a high sensitivity and strong specificity. The relative position of the PACAP38 and $\mathrm{NtA}$ structure is shown in Fig. 1A. In the present study, this method was employed for comparing the binding capacity with laminin between the PACAP38-NtA and wild-PACAP. The PACAP and recombinant protein, PACAP38-NtA, were diluted equally according to $0.5 / 64,0.5 / 16,0.5 / 4,0.5,8$ and $16 \mu \mathrm{M}$, and the binding capacity with laminin was detected, respectively. According to the absorbance value at OD450 nm, the laminin binding capacity with PACAP38-NtA was higher compared to PACAP at various concentrations (Fig. 2A). The detection results showed that the binding capacity of the two groups were significantly different $(\mathrm{P}<0.01)$, indicating that 

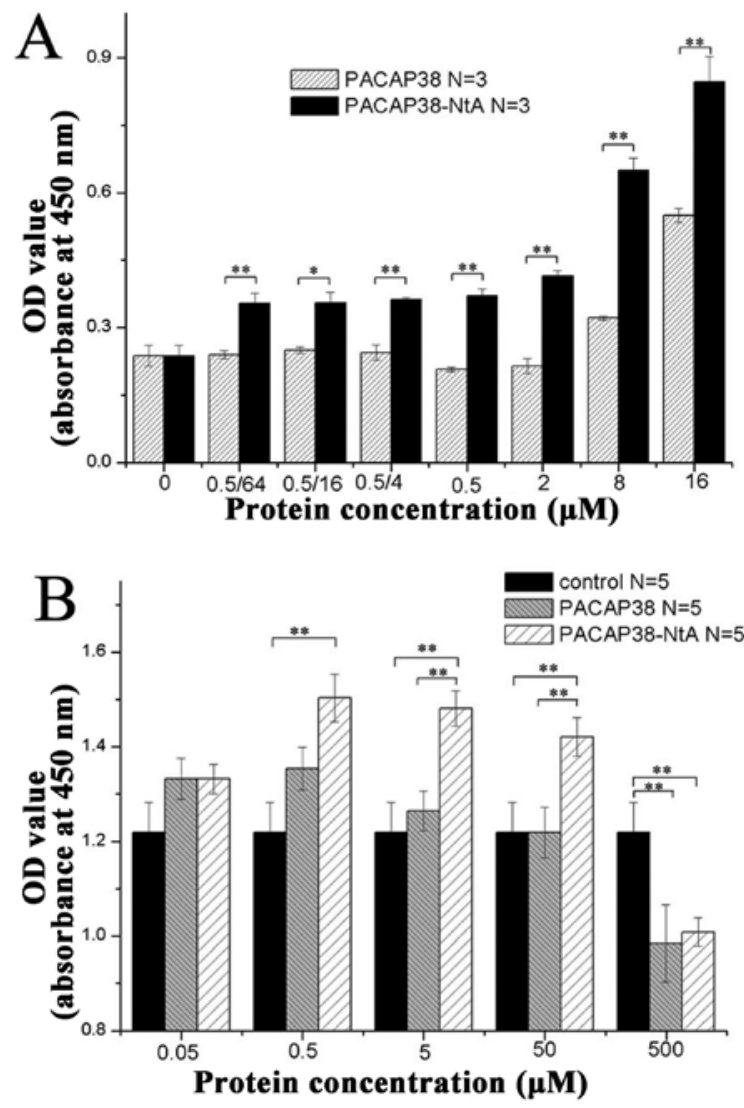

Figure 2. (A) Binding diagram of PACAP38-NtA and PACAP38 to laminin, measured by the ELISA assay [mean \pm standard deviation $(\mathrm{SD}) ; \mathrm{n}=5$ ]. (B) Bioactivity comparison of PACAP38-NtA and PACAP in vitro. Effect of PACAP38-NtA and PACAP on cell survival in PC12 cells by the CCK8 assay (mean $\pm \mathrm{SD} ; \mathrm{n}=6$ ). PACAP, pituitary adenylate cyclase-activating polypeptide; NtA, N-terminal agrin domain; IPTG, isopropylthiogalactoside; OD, optical density.

the recombinant protein, PACAP38-NtA, combined with the laminin protein. The C-terminal NtA of the PACAP38-NtA protein purified with molecular cloning maintains the original biological activity and it can target laminin binding.

With the CCK8 method, the antagonistic effect of the recombinant protein, PACAP38-NtA, and wild-type PACAP38 on the starved PC12 injury apoptosis was studied. The detection results are shown in Fig. 2B, from which it is clear that PACAP38-NtA is more efficient compared to wild-PACAP38 on the starved PC12 injury apoptosis when the protein concentration is within $0.05-50 \mathrm{nM}$, and the two proteins are strong on anti-apoptosis when the protein concentration is $0.5 \mathrm{nM}$. In addition, it was found that with the increase of the concentration, the antagonistic effect of recombinant protein PACAP38-NtA and wild-type PACAP38 on the starved PC12 injury apoptosis was weakened.

In the study, the promotion of the PC12 cell processes and growth was compared for PACAP38-NtA and wild-PACAP38 quantitatively, as shown in Fig. $3 \mathrm{~A}$ and B. In Fig. 3A, the density and length of the nerve processes across the transwell cell under the microscope after 3, 6, 12 and $24 \mathrm{~h}$ are shown; whereas in Fig. 3B, the absorbance value of the nerve processes across the transwell cell at $570 \mathrm{~nm}$ are shown subsequent to the addition of crystal violet to stain purple and dissolving by glacial acetic acid. PACAP38-NtA and PACAP38 promote the neuron differentiation, and there is no difference between the PACAP38-NtA and PACAP38 proteins.

Recovery time following corneal injury. The trephine injury method was applied for the construction of the corneal injury model $(35,36)$. Six C57 male mice were randomly divided into three groups; normal saline, $100 \mathrm{nM}$ PACAP38-NtA and $100 \mathrm{nM}$ PACAP38 groups. Subsequent to injuring the corneal epithelial cells in the left and right eyes of adult C57 mice with mini-keratome, $2 \%$ sodium fluorescein was applied for coloration (showing the injury area, Fig. 4A), and $5 \mu \mathrm{l}$ normal saline, $5 \mu 1100 \mathrm{nM}$ PACAP38-NtA and $5 \mu 1100 \mathrm{nM}$ PACAP38 were added, respectively. The repair process of the cornea in each group is shown in Fig. 4B. Recovery occurred in $18 \mathrm{~h}$ in the PACAP38-NtA group, which was significantly quicker compared to the control group with PACAP (24 h). The $100 \mathrm{nM}$ PACAP38 and control groups took less time compared to the blank control group in repairing the cornea $(36 \mathrm{~h}$ in the normal saline group). PACAP38-NtA promotes the corneal epithelial cell repairing, and its remediation effect is improved compared to wild-PACAP38 at a concentration of $100 \mathrm{nM}$.

\section{Discussion}

PACAP has neurotrophy and a neural restoration function. PACAP and its special receptor, PAC1, are widely spread in the peripheral nervous system, such as the cornea nerve $(29,37,38)$. According to these studies, PACAP can promote the repair of corneal epithelium and regulation of the inflammatory response of the ocular surface. Therefore, PACAP is a candidate polypeptide drug with broad application. However, according to clinical studies, PACAP through intravenous injection is decomposed by various enzymes in the blood, such as dipeptidyl peptidase (DPP)-IV and carboxypeptidase (CP), the half-life period is $3-10 \mathrm{~min}(7,39,40)$. Consequently, PACAP as the neural restoration drug for clinical treatment requires further study. Improving the clinical effect of the biological activity of PACAP and lengthening the action time of the biological activity would be a significant research direction for PACAP polypeptide drug development.

Using genetic engineering, two polypeptides with different functions can be connected together (Fig. 1A), but the biological activity of the two polypeptides mainly depends on whether the primary structure of the polypeptide alters the space structure of the active site region when folding into the space structure. In the present study, the expression vector of PACAP38-NtA was constructed with molecular cloning, and the active proteins were separated using a two-step purification method, with the purity $>90 \%$ (Fig. 1B-D). This polypeptide was identified as the amino acid sequence of PACAP38 through western blotting analysis (Fig. 1E), and it was preliminarily regarded as the polypeptide of interest.

The model of PC12 injury can be generated with numerous methods, such as $\mathrm{H}_{2} \mathrm{O}_{2}, \mathrm{MPP}^{+}$, glutamic acid and serum-free injuries. In the present study, the injury model of PC12 cell was created with serum-free injury, which can decrease the influence of serum on the experiment result, with a clear and stable effect. The anti-apoptosis experiment of CCK8 shows that the recombinant protein has the biological function of PACAP38, as it resists the PC12 cellular damage and apoptosis. Under the 

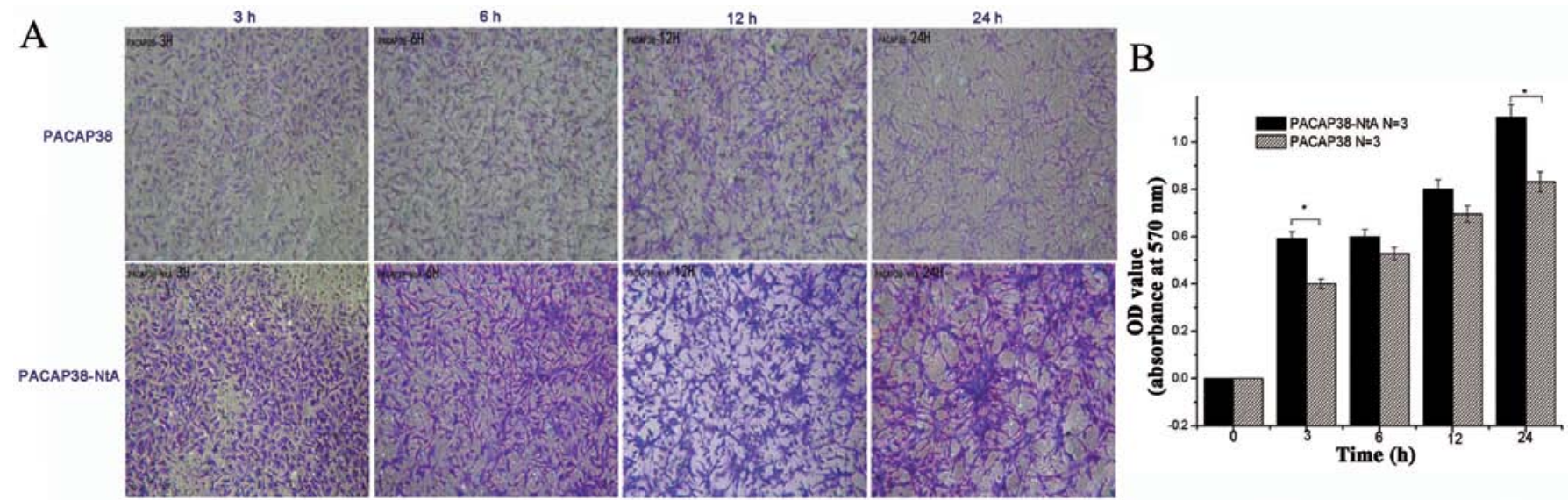

Figure 3. Effect of PACAP38-NtA and PACAP38 on neurite outgrowth in PC12 cells by NEO. (A) Transwell at $0,3,6,12$ and $24 \mathrm{~h}$ (magnification, 4x5). (B) Absorbance value (OD570) of PACAP38 and PACAP-NtA at 0, 3, 6, 12 and 24 h. PACAP, pituitary adenylate cyclase-activating polypeptide; $\mathrm{NtA}, \mathrm{N}$-terminal agrin domain; NEO, neurite outgrowth assay; OD, optical density.

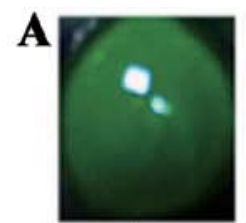

$\mathbf{O h}$

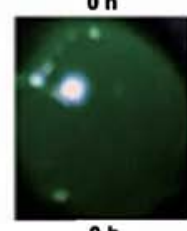

$\mathbf{O h}$

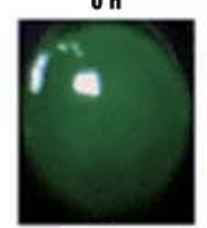

oh

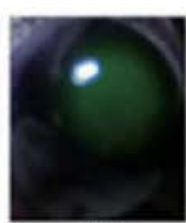

$12 \mathrm{~h}$

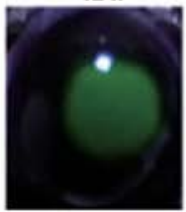

$12 \mathrm{~h}$

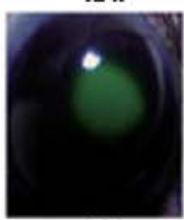

$12 \mathrm{~h}$

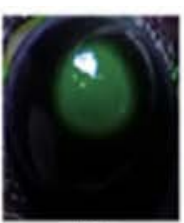

$18 \mathrm{~h}$

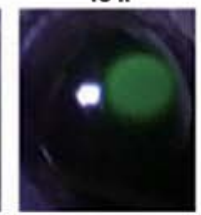

$18 \mathrm{~h}$

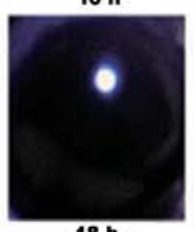

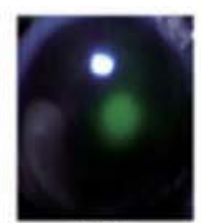

$24 \mathrm{~h}$

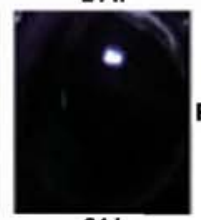

$24 \mathrm{~h}$

PACAP38-NtA
B

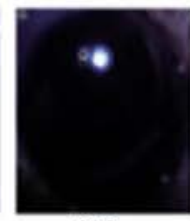

$36 \mathrm{~h}$

PACAP38

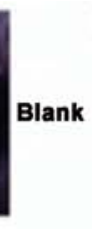

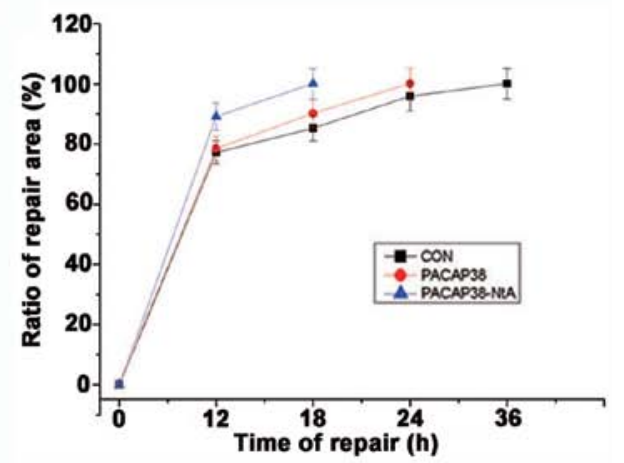

Figure 4. In vivo remediation effect with corneal injury model. (A) Injury area marked by sodium fluorescein. The concentration of PACAP38 and PACAP38-NtA was $100 \mathrm{nM}$ and the blank group was dropped with normal saline; $~ 5 \mu 1$ medicine and normal saline for each time-point. The repair times for PACAP38-NtA, PACAP38 and the control group were 18,24 and $36 \mathrm{~h}$, respectively. (B) The repair area changed with time. PACAP, pituitary adenylate cyclase-activating polypeptide; NtA, N-terminal agrin domain.

same concentration, the anti-apoptosis ability of the recombinant protein, PACAP38-NtA, was shown to be more efficient compared to PACAP38 (Fig. 2B). The experiment of the PC12 serum-free injury model has shown that the recombinant polypeptide also has the same in vitro biological activity of anti-nerve cell apoptosis as PACAP38.

According to the experiment, PACAP, as a neuropeptide, has the in vitro and in vivo biological function of promoting the growth of nervous processes. In the study, the biological activity of two types of polypeptide in promoting the growth of nervous processes has been compared through the in vitro experiments using the $\mathrm{PC} 12$ nerve regeneration quantitative analysis. The nervous processes of PC12 cell were detected according to the quantitative determination reported by Smit et al (34), and quantitative analysis was performed for 3, 6, 12 and $24 \mathrm{~h}$ after PACAP38 and PACAP38-NtA were added. According to the results, the recombinant polypeptide, PACAP38-NtA, promotes the growth of PC12 cellular processes, and the ability of the recombinant polypeptide in promoting the growth of the nervous processes at different time points is more efficient compared to the wild-polypeptide PACAP38.

PACAP promotes the growth of trigeminal cells in the corneal injury, as well as the restoration of the epithelial cell and lacrimal gland in laser-assisted in situ keratomileusis rabbit corneal surgery. The mechanism may be that it can promote the trigeminal cell to secrete active neurotransmitters to enhance the proliferation, differentiation and production of collagen VII. In the present study, the mechanically-injured corneal epithelial cells of C57 mice were adopted for the model, and $2 \%$ sodium fluorescein staining directly exhibited the injured section. The size of the stained section can distinguish the remediation effect of the polypeptide at the same moment. The effect of repairing the injured corneal epithelial cells with the recombinant polypeptide, PACAP38-NtA, and wild-PACAP38 were studied with the mechanically damaged 
cornea C57 mouse model. According to the experiment, under the in vivo conditions, it takes $18 \mathrm{~h}$ for $100 \mathrm{nM}$ of the PACAP38-NtA polypeptide to repair the injured corneal epithelial cell, while it takes $24 \mathrm{~h}$ for $100 \mathrm{nM}$ of the PACAP38 polypeptide. Evidently, it accelerates the process of repairing the injured corneal epithelial cells of C57 mice.

According to previous studies, once the nerve is damaged mechanically, Schwann cells will gather in the injured section and secrete substantial laminin $(16,22)$. The polypeptide PACAP-NtA constructed in the present study has the biological function of combining with laminin, and as a result, it can anchor near the laminin of the injured nerve section, with a significant advantage in repairing the injured nerve. The ELISA experiment of the recombinant protein, PACAP38-NtA, and laminin has shown that the recombinant protein can combine with laminin when it reaches the nanomole and micromole concentration level, with an evident comparative difference from the negative docking of PACAP38. Consequently, it can be predicted that the recombinant protein PACAP38-NtA has the biological activity of $\mathrm{NtA}$, as it can combine with laminin. The combination of the recombinant protein, PACAP38-NtA, and laminin can gather in the injured section and militate constantly, allowing the neural restoration function of PACAP38.

In conclusion, the recombinant protein, PACAP-NtA, has been constructed, expressed and purified with the genetic engineering method, and according to the experiment, the recombinant protein is equipped with such biological functions as preventing the apoptosis of injured nerve and promoting the growth of nervous processes of the PACAP38 polypeptide. In addition, the polypeptide can anchor on the laminin of the injured section of the nerve, and improve the utilization efficiency of the PACAP38 polypeptide by the injured section. The recombinant polypeptide may become a novel type of candidate drug for promoting the restoration of injured nerve, and further studies are required.

\section{Acknowledgements}

The present study was supported by the Science and Technology Project of Guangzhou (grant no. 2011J4300107).

\section{References}

1. Miyata A, Arimura A, Dahl RR, et al: Isolation of a novel 38 residue-hypothalamic polypeptide which stimulates adenylate cyclase in pituitary cells. Biochem Biophys Res Commun 164 567-574, 1989.

2. Wray V, Kakoschke C, Nokihara K and Naruse S: Solution structure of pituitary adenylate cyclase activating polypeptide by nuclear magnetic resonance spectroscopy. Biochemistry 32 : 5832-5841, 1993.

3. Jüppner H, Schipani E, Bringhurst FR, et al: The extracellular amino-terminal region of the parathyroid hormone (PTH)/PTH-related peptide receptor determines the binding affinity for carboxyl-terminal fragments of PTH (1-34). Endocrinology 134: 879-884, 1994.

4. Wei Y and Mojsov S: Tissue specific expression of different human receptor types for pituitary adenylate cyclase activating polypeptide and vasoactive intestinal polypeptide: implications for their role in human physiology. J Neuroendocrinol 8: 811-817, 1996.

5. Gourlet P, Vandermeers A, Robberecht P and Deschodt-Lanckman M: Vasoactive intestinal peptide (VIP) and pituitary adenylate cyclase-activating peptide (PACAP-27, but not PACAP-38) degradation by the neutral endopeptidase EC 3.4.24.11. Biochem Pharmacol 54: 509-515, 1997.
6. Ohkubo S, Kimura C, Ogi K, et al: Primary structure and characterization of the precursor to human pituitary adenylate cyclase activating polypeptide. DNA Cell Biol 11: 21-30, 1992.

7. Vaudry D, Gonzalez BJ, Basille M, Yon L, Fournier A and Vaudry H: Pituitary adenylate cyclase-activating polypeptide and its receptors: from structure to functions. Pharmacol Rev 52: 269-324, 2000.

8. Inooka $\mathrm{H}$, Ohtaki $\mathrm{T}$, Kitahara $\mathrm{O}$, et al: Conformation of a peptide ligand bound to its G-protein coupled receptor. Nat Struct Biol 8: 161-165, 2001.

9. Shimizu N, Guo J and Gardella TJ: Parathyroid hormone (PTH)-(1-14) and -(1-11) analogs conformationally constrained by alpha-aminoisobutyric acid mediate full agonist responses via the juxtamembrane region of the PTH-1 receptor. J Biol Chem 276: 49003-49012, 2001.

10. Tibaduiza EC, Chen C and Beinborn M: A small molecule ligand of the glucagon-like peptide 1 receptor targets its amino-terminal hormone binding domain. J Biol Chem 276: 37787-37793, 2001.

11. Miyata A, Jiang L, Dahl RD, et al: Isolation of a neuropeptide corresponding to the N-terminal 27 residues of the pituitary adenylate cyclase activating polypeptide with 38 residues (PACAP38). Biochem Biophy Res Commun 170: 643-648, 1990.

12. Inooka $H$ and Shirakawa $M$ : Conformation of a peptide ligand bound to its G-protein coupled receptor and its implication for ligand transportation. Tanpakushitsu Kakusan Koso 47: 787-793, 2002 (In Japanese).

13. Laburthe M and Couvineau A: Molecular pharmacology and structure of VPAC Receptors for VIP and PACAP. Regul Pept 108: 165-173, 2002.

14. Laburthe M, Couvineau A and Marie JC: VPAC receptors for VIP and PACAP. Receptors Channels 8: 137-153, 2002.

15. Runge S, Wulff BS, Madsen K, Brauner-Osborne $\mathrm{H}$ and Knudsen LB: Different domains of the glucagon and glucagon-like peptide-1 receptors provide the critical determinants of ligand selectivity. Br J Pharmacol 138: 787-794, 2003.

16. Shioda S, Shuto Y, Somogyvari-Vigh A, et al: Localization and gene expression of the receptor for pituitary adenylate cyclase-activating polypeptide in the rat brain. Neurosci Res 28 : 345-354, 1997.

17. Nowak JZ and Zawilska JB: PACAP in avians: origin, occurrence, and receptors-pharmacological and functional considerations. Curr Pharm Des 9: 467-481, 2003.

18. Bourgault S, Vaudry D, Segalas-Milazzo I, et al: Molecular and conformational determinants of pituitary adenylate cyclase-activating polypeptide (PACAP) for activation of the PAC1 receptor. J Med Chem 52: 3308-3316, 2009.

19. Linden A, Hansson L, Andersson A, et al: Bronchodilation by an inhaled VPAC (2) receptor agonist in patients with stable asthma. Thorax 58: 217-221, 2003.

20. Yuhara A, Nishio C, Abiru Y, Hatanaka H and Takei N: PACAP has a neurotrophic effect on cultured basal forebrain cholinergic neurons from adult rats. Brain Res Dev Brain Res 131: 41-45, 2001.

21. Laburthe M, Couvineau A and Tan V: Class II G protein-coupled receptors for VIP and PACAP: structure, models of activation and pharmacology. Peptides 28: 1631-1639, 2007.

22. Hautmann M, Friis UG, Desch M, et al: Pituitary adenylate cyclase-activating polypeptide stimulates renin secretion via activation of PAC1 receptors. J Am Soc Nephrol 18: 1150-1156, 2007.

23. Cazillis M, Gonzalez BJ, Billardon C, et al: VIP and PACAP induce selective neuronal differentiation of mouse embryonic stem cells. Eur J Neurosci 19: 798-808, 2004.

24. Zhang H, Yu R, Liu X, Guo X and Zeng Z: The expression of $\mathrm{PAC} 1$ increases in the degenerative thymus and low dose PACAP protects female mice from cyclophosphamide induced thymus atrophy. Peptides 38: 337-343, 2012.

25. Harmar AJ, Fahrenkrug J, Gozes I, et al: Pharmacology and functions of receptors for vasoactive intestinal peptide and pituitary adenylate cyclase-activating polypeptide: IUPHAR review 1. B J Pharmacol 166: 4-17, 2012.

26. Racz B, Gasz B, Borsiczky B, et al: Protective effects of pituitary adenylate cyclase activating polypeptide in endothelial cells against oxidative stress-induced apoptosis. Gen Comp Endocrinol 153: 115-123, 2007.

27. Horvath G, Brubel R, Kovacs K, et al: Effects of PACAP on oxidative stress-induced cell death in rat kidney and human hepatocyte cells. J Mol Neurosci 43: 67-75, 2011. 
28. Horvath G, Reglodi D, Opper B, et al: Effects of PACAP on the oxidative stress-induced cell death in chicken pinealocytes is influenced by the phase of the circadian clock. Neurosci Lett 484: $148-152,2010$.

29. Fukiage C, Nakajima T, Takayama Y, Minagawa Y, Shearer TR and Azuma M: PACAP induces neurite outgrowth in cultured trigeminal ganglion cells and recovery of corneal sensitivity after flap surgery in rabbits. Am J Ophthalmol 143: 255-262, 2007.

30. Wang ZY, Alm P and Hakanson R: Distribution and effects of pituitary adenylate cyclase-activating peptide in the rabbit eye. Neuroscience 69: 297-308, 1995.

31. Fu SY and Gordon T: The cellular and molecular basis of peripheral nerve regeneration. Mol Neurobiol 14: 67-116, 1997.

32. Daggett DF, Cohen MW, Stone D, Nikolics K, Rauvala H and Peng HB: The role of an agrin-growth factor interaction in ACh receptor clustering. Mol Cell Neurosci 8: 272-285, 1996.

33. Sun W, Sun C, Zhao H, et al: Improvement of sciatic nerve regeneration using laminin-binding human NGF-beta. PloS One 4 e6180, 2009.

34. Smit M, Leng J and Klemke RL: Assay for neurite outgrowth quantification. Biotechniques 35: 254-256, 2003.
35. Li Z, Burns AR, Han L, Rumbaut RE and Smith CW: IL-17 and VEGF are necessary for efficient corneal nerve regeneration. Am J Pathol 178: 1106-1116, 2011.

36. Lee SJ, Kim JK, Seo KY, Kim EK and Lee HK: Comparison of corneal nerve regeneration and sensitivity between LASIK and laser epithelial keratomileusis (LASEK). Am J Ophthalmol 141: 1009-1015, 2006.

37. Waschek JA: Multiple actions of pituitary adenylyl cyclase activating peptide in nervous system development and regeneration. Dev Neurosci 24: 14-23, 2002.

38. Somogyvári-Vigh A and Reglodi D: Pituitary adenylate cyclase activating polypeptide: a potential neuroprotective peptide. Curr Pharma Des 10: 2861-2889, 2004.

39. Li M, Maderdrut JL, Lertora JJ and Batuman V: Intravenous infusion of pituitary adenylate cyclase-activating polypeptide (PACAP) in a patient with multiple myeloma and myeloma kidney: a case study. Peptides 28: 1891-1895, 2007.

40. Mentlein R: Dipeptidyl-peptidase IV (CD26) - role in the inactivation of regulatory peptides. Regul Pept 85: 9-24, 1999. 